\title{
Editorial
}

\section{Cardiac surgery in elderly patients: benefits and resource priorities}

Any definition of old age is arbitrary and influenced by several factors, but cultural background and national retirement policies are often dominant. The so called "elderly" are a heterogeneous population whose age span covers three decades or more. The over 65 age group in England and Wales are projected to increase by $8 \%$ for the period 1991-2031, ${ }^{1}$ but over this 40 year span the projected increase in people aged $75-84$ is almost $50 \%$. The prevalence of disability, particularly from cardiovascular disease, and multiple morbidity rises with age. More positively, chronic disability in older people has been falling over the past decade and this process is likely to continue. ${ }^{2}$

Since the middle of the 1980s developments in the surgical management of cardiovascular disease, especially for aortic valve disease, has had a dramatic impact on quality of life and prognosis at all ages. Symptomatic cardiac disease in elderly patients is often more refractory to continued medical measures than in younger adults, and surgery may be the only option to restore functional competence and quality of life.

\section{Is cardiac surgery in old age justified by outcome measures?}

For any medical or surgical intervention we must know if "added years (are) onus or bonus". ${ }^{3}$ What supporting evidence is there that cardiac surgery, particularly for aortic valve disease, or combined coronary artery bypass grafting (CABG) and valve replacement, improves quality of life with an acceptable (to the patient) surgical risk? A consensus on cardiac surgical preoperative and postoperative mortality and morbidity in old people is difficult to establish from much of the reported literature, as case mix, type of surgical procedure, and preoperative and postoperative care systems often differ considerably.

Patients $>80$ years have a higher mortality than those a decade younger. ${ }^{4}$ In unselected patients undergoing a range of major cardiac surgical procedures the 30 day postoperative mortality rate is $6.8 \%$ in patients $>70$ years compared with $2.5 \%$ in younger patients, ${ }^{4}$ which is not excessive. Indeed this is a remarkable achievement considering that over one third of old people presenting for cardiac surgery have severe disease. Preoperative pulmonary hypertension and reduced left ventricular function, ${ }^{5}$ preoperative intensive care admission, ${ }^{6}$ and emergency surgery ${ }^{7}$ are markers of potential postoperative fatality and complications, and the combination of CABG and aortic valve replacement is associated with a higher mortality than for a single procedure, ${ }^{5}$ but not invariably so. ${ }^{8}$ Comorbid conditions, particularly impaired renal function and peripheral vascular disease, increase postoperative risks. ${ }^{8}$ This highlights the urgency of improving current medical practice to ensure early detection, and competent medical management of co-existing disease. The actuarial survival 5-7 years after major cardiac surgery is comparable to the (age and sex matched) general population. ${ }^{58}$ Despite acceptable survival data the fundamental issue is long term improvement of function and quality of life. Symptom control is greatly improved following aortic valve surgery in seriously compromised patients compared with patients on continued medical treatment. ${ }^{9}$ Aortic valve surgery improves New York Heart Association functional class by a median of two classes, ${ }^{58}$ and measures of independent living and satisfaction with quality of life are substantially improved as patients continue to live in their own homes. $^{71011}$

\section{In-hospital costs of cardiac surgery in elderly patients}

The in-hospital costs for cardiac surgery are greater for very elderly patients than for younger patients, and may exceed locally determined contract prices in the UK. ${ }^{8}$ However, one may question how realistically contract prices reflect the demographic pattern of UK society.

Reduction of cardiac surgical cost in the very old may require new strategies with greater multidisciplinary involvement, earlier referral to cardiac surgery, and more sophisticated identification of critical risk factors. Variable life-adjusted display monitoring enhances surgical self assessment and outcome. ${ }^{12}$ Wider use of preoperative $\beta$ blockers, as shown in non-cardiac surgery, ${ }^{13}$ may reduce mortality in elderly patients requiring urgent valve surgery but who are too unwell for CABG. Further developments of non-invasive coronary artery positron emission tomography scanning and intravenous ultrasound guided angioplasty and stent monitoring may permit two stage intervention of vulnerable patients and may in future improve valve surgery outcomes. Earlier detection and optimum management of cardiac disease and comorbid conditions may significantly reduce the duration of stay in cardiac recovery and intensive care beds.

\section{Rationing and cardiac surgery in the elderly}

It has been suggested that focusing on more narrow aspects of health resource priorities is easier. ${ }^{14}$ It is beyond dispute that priorities in the delivery of health resources require urgent reappraisal. Calculations incorporating Dutch insurance data estimate that $30-40 \%$ of health related costs may be incurred during the last 18 months of life and may account for much of the inevitable increase in these costs in old age. ${ }^{15}$ Moreover, a substantial segment of UK National Health Service resources are not applicable to older people, and at the other extreme of life huge costs are generated by procedures that have dubious outcome benefit. Are these wider issues to remain undisturbed because they are too difficult to consider? Too difficult for whom? Cost of cardiac surgery is likely to be high on the agenda of any debate on rationing, ${ }^{14}$ and older people may be seen as less deserving of expensive resources, a viewpoint based on a fallacy that the wealth of a country is derived from those currently working. Wealth creation is built on a heritage of skills, knowledge, and processes developed by countless past generations, and the young and old are equal heirs to this inheritance.

Unrelieved disability has both a human and a financial cost. Discounting human costs, a popular past time among holders of the public purse, dependency in older people commonly requires continuing support from social service agencies or more frequent admission to nursing home care 
at a cost of approximately $£ 20000$ (US\$32 000) per year. Thus, separate budget holding for health and social services distorts the true cost of the conservative versus surgical intervention equation.

\section{Conclusion}

The evidence is compelling that after aortic valve surgery (with or without CABG) most elderly survivors are free from disabling symptoms and are enabled to continue to live at home with a level of independence comparable to the general elderly population. Given the stark and emotional choice of lifesaving cardiac surgery for young or old it is of course relatively easy to influence society to opt for the former. Is this equitable or ethical? Indeed, will judgment really be based on sound economical appraisal of a wide range of factors in the health and social fields? Maybe a broader remit may decide that lifesaving cardiac surgery for all ages has a higher priority than other NHS services.

Professor Emeritus, University of Wales,

M S JOHN PATHY

Research Director, Health Care Research Unit,

St Woolos Hospital, Newport,

South Wales NP9 45Z, UK

1 Medical Research Council. The health of the UK's elderly people. MRC topic review. London: Medical Research Council, 1994.
2 Manton KG, Stalland E, Corder L. Changes in morbidity and chronic disability in the US elderly population: evidence form the 1982, 1984 and 1989 national long-term care survey. F Gerontol 1995;50:5104-204.

3 Bonita R. Added years, onus or bonus? Lancet 1997;350:1167-8.

4 Zaidi AM, Fizpatrick AP, Kenan DJ, et al. Good outcomes from cardiac surgery in the over 70s. Heart 1999;82:134-7.

5 Kirsch $\mathrm{M}$, Guesnier $\mathrm{L}$ LeBesnerais $\mathrm{P}$, et al. Cardiac operations in octogenerians: perioperative risk factors for death and impaired autonomy. Ann Thorac Surg 1998:66:60-7.

6 Deiwick M, Tandler R, Mollhof T, et al. Heart surgery in patients aged eighty years and above: determinants of morbidity and mortality. Thorac Cardiovasc Surg 1997;45:119-26.

7 Chocron S, Rude N, Dussaucy A, et al. Quality of life after open heart surgery in patients over 75 years old. Age Ageing 1996;25:8-11.

8 Gilbert T, Orr W, Banning A. Surgery for aortic stenosis in severely symptomatic patients older than 80 years of age: experience in a single UK centre. Heart 1999;82:138-42

9 Bouma BJ, van den Brink RBA, van den Meulen JHP. To operate or not on elderly patients with aortic stenosis: the decision and its consequences. Heart 1999;82:143-8.

10 Khan JH, McElhinney DB, Hall TS, et al. Cardiac valve surgery in octogenarians: improving quality of life and functional status. Arch Surg 1998;133:887-93.

11 Kumar P, Zehr KJ Chang A, et al. Quality of life in octagenerians after open heart surgery. Chest 1995;108:919-26.

12 Lovegrove J, Valencial O, Treasure T, et al. Monitoring the results of cardiac surgery by variable life-adjusted display. Lancet 1997;350:1128-30.

13 Mangano DT, Layung EL, Wallace A, et al, for the Multicenter Study of Preoperative Ischemia Research Group. Effect of atenolol on mortality and cardiovascular morbidity after non-cardiac surgery. $N$ Engl $f$ Med cardiovascular mo

14 Klein R. Puzzling out priorities: why we must acknowledge that rationing is a political process [editorial]. BMF 1998;317:959-60.

15 van Weel C, Michels J. Dying, not old age, to blame for costs of health care. Lancet 1997;350:1159-60. 